\title{
Das Konzept der „Sakralität der Person“: ethische Verpflichtung zum Schutz der Schöpfung oder anthropozentrische Hybris?
}

Jakob Ohm

Wenn man in einem tierethischen Kontext ein Konzept zu rezipieren versucht, das explizit auf den Menschen hin ausgelegt ist und noch dazu in einer recht klassischen Formulierung über den Menschen als Person spricht, so mag der erste Eindruck Verwunderung sein. Dies mag vielleicht durch den schon im Titel meines Beitrages erscheinenden zweiten Begriff der Sakralität noch gesteigert werden. So gelten beide Termini, der der Person wie der des Heiligen, möglicherweise als derart durch den Ballast ihrer wechselnden Bedeutungsebenen durch die Jahrhunderte als verbraucht und wenig anschlussfähig für eine - leider - in der katholischen Theologie viel zu junge Disziplin wie die Tierethik, die doch vielleicht nach unverbrauchten und weniger sperrigen Vokabeln sucht, um ihr berechtigtes Anliegen zu transportieren. Umso größer mag vielleicht das Erstaunen oder das Unverständnis sein, wenn ein viel diskutierter Denker wie Hans Joas den Versuch unternimmt, eine große Meistererzählung wie die der Sakralisierung der Person anzubieten, ohne auch nur einen einzigen Blick auf die Conditio des nicht-menschlichen Lebens zu wagen. Folgende Fragen drängen sich auf: Setzt sich in diesem Konzept die jahrhundertealte Tradition der „Tiervergessenheit“" in der Ethik fort? Wird hier vielleicht jener unheilvolle Rechtfertigungszusammenhang von Theologie und (ungerechten) Machtstrukturen fortgesetzt, den Kurt Remele in Anschluss an Matthew Lamb und Gregory Baum als "Sakralismus“ bezeichnet (Kurt Remele 2019, 53)? Und darf ein solches Konzept überhaupt in einer theologischen Ethik rezipiert werden, die sich fernhalten möchte von einer Hybris, die den Menschen als konsumierenden „Interplanetaris Predator" (Rainer Hagencord ${ }^{4} 2009$, 43), den nur auf sich selbst verengten Unterwerfer des Kosmos, zum einzigen Inhalt ihrer Reflexion macht? Ich möchte die Antwort vorwegnehmen: Meines Erachtens nach birgt die Rede von der menschlichen Person als dem Sakralen durchaus das Potenzial, um eine Schieflage in der Mensch-Tier-Beziehung zu korrigieren. Dies kann jedoch nur intellektuell verantwortet werden, wenn die Vermittlung der Begriffe Person und Sakralität, wie sie bei Hans Joas stattfindet, zunächst näher untersucht 
wird und schließlich dem nicht nur wissenschaftlichen, sondern auch sehr lebenspraktischen Testfall der Begegnung mit dem Tier als „Mitgeschöpf“ (Fritz Blanke 1993, 9-13), also als Teil der moralischen Ordnung, das um seiner selbst willen in die ethische Güterabwägung mit einbezogen wird (Eberhard Schockenhoff 2009, 564), ausgesetzt wird.

In meinem Beitrag möchte ich daher untersuchen, inwieweit aus Sicht der theologischen Ethik eine Vermittlung der eminent theologischen Begriffe Sakralität und Person zu einem tieferen Verständnis der Stellung des Menschen im Zusammenleben mit anderen Geschöpfen beitragen kann. Dieser Frage möchte ich im Verlauf meiner Überlegungen in folgenden drei Schritten nachgehen:

Zunächst soll ein kurzer Überblick über einige anthropologische $\mathrm{He}$ rausforderungen der Gegenwart gegeben werden, die die Dringlichkeit einer Neuartikulation des christlichen Personalismus verständlicher machen und an denen aufgezeigt werden kann, dass Mensch und Tier ähnlichen Bedrohungsszenarien ausgesetzt sind, die es wissenschaftlich zu durchdringen gilt.

Anschließend soll in einem zweiten Schritt eine kritische Begriffsklärung folgen, wie die Rede von der Person und dem Sakralen bei Hans Joas zu verstehen ist und wie deren Vermittlung erfolgt. Dazu werde ich die Grundzüge des Konzeptes bei Hans Joas, sofern sie für das Thema dieses Beitrages von Bedeutung sind, in der gebotenen Kürze nachzeichnen.

Abschließend möchte ich darlegen, weshalb das Konzept von Hans Joas meiner Meinung nach trotz seiner vermeintlichen Tiervergessenheit keinen unterbewussten Vorschub für eine anthropozentrische Hybris des Menschen in ethischen Fragestellungen leistet. Vielmehr möchte ich einen Ausblick wagen auf eine Weiterentwicklung des Modells von Hans Joas, die weiterhin die Rede von der Person als dem Heiligen ermöglicht, jedoch in eine schöpfungstheologisch begründete Weite führt, die andere Lebewesen in ihrer Andersheit wertschätzt und diese nicht nur aus Eigeninteresse zu bewahren sucht, wie es etwa bei Immanuel Kant beschrieben wird, der nur Personen den Wert zugesteht, als Zweck an sich zu gelten (Immanuel Kant $\left.{ }^{4} 1982,107\right)$.

\section{Reduktionistische Menschenbilder als Herausforderung auch für die Tierethik}

Der in Oxford lehrende Philosoph Larry Siedentop hat in seinem Werk Inventing the Individual eindrücklich herausgestellt, dass die liberale Gesellschaftsordnung westlicher Prägung vor allem auf einer durch das Christentum initiierten moralischen Revolution beruht, welche durch zwei Kernele- 
mente gekennzeichnet ist (Larry Siedentop 2014, 50-100): Zum einen wird der Mensch als ein Wesen mit „moralischer Tiefe“ (Larry Siedentop 2014, 67) im Sinne einer sittlichen Kompetenz verstanden, der als Einzelner in der Lage ist, freie ethisch relevante Entscheidungen zu treffen und nicht in Gänze durch übergeordnete Größen wie etwa die Familie (oder einen Pater familias) oder den Staat (oder die antike Polis) moralisch determiniert wird. Zum anderen wurde durch die christliche Theologie, wie sie vor allem von Paulus von Tarsus vertreten wurde, eine moralische Gleichheit hergestellt. Dies bedeutet, dass der Mensch durch seine Personalität in den Stand freier ethischer Wahl versetzt wird und nicht etwa durch seine soziale Rolle oder seine Stellung im Gemeinwesen erst ein moralisches Subjekt werden muss (Larry Siedentop 2014, 67-79). Diese Analyse regt zu einer sensiblen Wahrnehmung für gesellschaftliche Tendenzen und Akteure an, die Menschenbilder anbieten oder befördern, die im Vergleich mit der oben genannten "Anthropologie der Tiefe" im Sinne einer sittlichen Kompetenz, die im Forum internum des Menschen angesiedelt ist, als reduktionistisch zu kennzeichnen sind. Beispielsweise stellt die rasante Entwicklung der Künstlichen Intelligenz (KI) und der Robotik die klassische Lehre von der Personalität des Menschen unter neuen Rechtfertigungsdruck und stellt die Frage nach dem genuin „Menschlichen“ des Menschen in größter Dringlichkeit, um der Gefahr des „Maschinenmenschen“ (Thomas Bauer 2018, 94), der sich für den kapitalistischen Produktionsprozess immer weiter selbst optimiert, als neuem Leitmotiv des menschlichen Selbstverständnisses intellektuell zu begegnen. Noch drängender scheinen jedoch gesellschaftliche Entwicklungen zu sein, die den Menschen nur nach einigen seiner sozialen Rollen zu definieren versuchen, wie z. B. der Rolle des Konsumenten, was die Gefahr in sich birgt, zu einem „techno-ökonomischen Paradigma“ (Peter Turkson 2019, 9) zu führen. Konsumismus und Materialismus, die den Menschen allein auf seine Rolle auf dem Markt reduzieren, stehen im Mittelpunkt der ethischen Debatte nicht nur innerhalb der akademischen Theologie, sondern auch des kirchlichen Lehramtes insbesondere unter dem Pontifikat von Papst Franziskus (Laudato si' [LS] 43-47). Vor diesem nachdenklich stimmenden Befund scheint das Anliegen von Hans Joas, nämlich die „Neuartikulation des christlichen Personalismus“ (Hans Joas 2012, 208), nur allzu gut verständlich. Jedoch muss kritisch angemerkt werden, dass das christliche Bild vom

1 Vgl. zur Anthropologie der „Tiefe“ über Siedentop hinaus auch die Ausführungen von Clemens Sedmak zum „Primat der tiefen Person“ in der katholischen Soziallehre (Clemens Sedmak 2017, 67-69). 
Menschen als Person nicht nur für ethische Probleme im Bereich des Menschlichen sensibilisiert, sondern auch auf Problemstellungen aufmerksam machen kann, die mit dem Verhältnis des Menschen zum Tier zu tun haben und die aus den oben genannten Krisenphänomenen resultieren. Dass Tiere verzweckt, objektiviert und nur unter den Gesichtspunkten von Wirtschaftlichkeit und Nützlichkeit beurteilt werden, ist zwar in der Tat kein Phänomen allein unserer Tage. Wenn jedoch das Verständnis des Menschen als Wesen mit „moralischer Tiefe“, welches mit der Freiheit ausgestattet ist, seine sittliche Vernunft zur guten Tat hin auszurichten, immer weiter erodiert, dann ist es mehr als wahrscheinlich, dass sich dieser Wandel im Selbstbild des Menschen auch auf sein Verhältnis zu seiner natürlichen Umwelt und zur Tierwelt im Besonderen auswirkt. Dass Papst Franziskus in seiner Lehrverkündigung derzeit einen besonderen Fokus auf ökonomisch motivierte Enthumanisierungstendenzen legt und diese auch mit dem Wohl der Tiere zusammendenkt (LS 92), kann so interpretiert werden, dass auch die offizielle Soziallehre der katholischen Kirche tierethische Debatten in den Blick nimmt, was jedoch immer unter dem Maßstab der Personalität des Menschen geschieht, was im letzten Abschnitt dieses Beitrags nochmal kritisch aufgenommen werden soll. Die hier nur sehr kurz skizzierte Tendenz zu reduktionistischen Menschenbildern in der ökonomischen und geistigen Alltagskultur, aber auch in akademischen Debatten wie etwa derjenigen um den Transhumanismus, soll an dieser Stelle nur die Sensibilität für die Schnittstelle zwischen einer personalen Ethik und der Tierethik erhöhen. Denn eine dem kalten technokratischen Paradigma unterworfene Lebenswirklichkeit, in der „keine tierische Wärme vorhanden ist und kein heißes Blut kreist" (Nikolai A. Berdiajew 2018, 83), ist ein Bedrohungsszenario nicht nur für den Menschen, sondern für jedwedes Leben. Der weitere Gedankengang wird sich folglich an dieser Schnittstelle unter der Leitfrage bewegen, inwiefern eine Ethik der Person, die nicht auf die Stellung des Menschen im Produktionsprozess abhebt, sondern auf den Menschen als Wesen mit "moralischer Tiefe“ angelegt ist, auch Implikationen zum Umgang mit Tieren in sich birgt, insofern sie letztlich auf eine gemeinsame schöpfungstheologische Ursprünglichkeit rekurriert.

\section{Die Vermittlung zwischen Personalität und Sakralität bei Hans Joas - ein Ansatzpunkt für eine personale Ethik im Umgang mit Tieren?}

Im Untertitel seines 2011 erschienenen Buches Die Sakralität der Person nennt Hans Joas die Intention, die er mit seiner Theorie der Sakralisierung 
der Person verbindet, nämlich eine „neue Genealogie der Menschenrechte" anzubieten. Dieses Bestreben reiht sich in Hans Joas' bisheriges Arbeitsfeld der Erforschung des Ursprungs der Werte ein (Hans Joas 1997). Was veranlasst Hans Joas aber dazu, einen neuen Versuch der Erforschung der Ursprünge der Menschenrechte zu unternehmen, wo doch die Fülle der Literatur zu diesem Thema darauf hinzuweisen scheint, dass der Forschungsstand bereits weit fortgeschritten ist? Laut Hans Joas lassen sich die Argumentationslinien für die Kodifizierung der Menschenrechte nach der amerikanischen und der französischen Revolution in zwei gängigen Narrativen zusammenfassen (Hans Joas 2011, 23-63):

1. Zum einen die aufklärerisch-humanistische Erzählung: Hier sieht Hans Joas eine Argumentationslinie, die versucht, eine Erzählung zu entwickeln, die die Menschenrechtserklärungen in erster Linie als Produkt eines Emanzipationsprozesses des Individuums gegen eine christliche Ideologie versteht, die den Menschen in seiner Gesamtheit erfassen und moralisch bevormunden wollte. Im Gegensatz dazu stellt sich der Revolutionär im Pathos der aufklärerischen Selbstbefreiung gegen das kirchliche Gewissensdiktat und die unglückliche Verstrickung von Krone und Altar und gibt sich und seinen Mitbürgern eine kodifizierte Freiheitscharta, nämlich die Menschenrechtserklärung. In der Artikulation der Menschenrechtserklärung in der aufklärerischen Tradition spricht Hans Joas in Anlehnung an Max Weber von einer "charismatischen Verklärung der Vernunft" (Hans Joas 2011, 61).

2. Zum anderen identifiziert Hans Joas als zweite Erzählung das christlich-jüdische Menschenbild, wie es sich aus den Evangelien ableiten lässt (Hans Joas 2011, 204-251). Doch sei hier nur an die antimodernistischen Tendenzen der römischen Kurie zu erinnern, die in der Kodifizierung der Menschenrechte nur eine unliebsame historische Entwicklung sah, die es eigentlich nicht hätte geben dürfen, nämlich die Loslösung des Menschen von der moralischen Autorität des kirchlichen Lehramtes. Mit anderen Worten: Hans Joas ist sehr skeptisch, wenn Theologen versuchen, die Menschenrechte als eine notwendige Entwicklung des Christentums darzustellen und die Menschenrechte nur auf dieser Grundlage zu rechtfertigen, so wie es teilweise in theologischen Ansätzen nach den verheerenden Gewalterfahrungen zweier Weltkriege und dann vor allem nach dem II. Vatikanischen Konzil geschah.

In den beiden genannten Erzähltraditionen sieht Hans Joas eine Verengung des Denkens, die historisch falsch zu sein scheint (Hans Joas 2013, 15). Einerseits verkennt der aufklärerische Blick auf den historischen Prozess die Rolle des Christentums in der Ideengeschichte von Menschenwürde und Menschenrechten. Andererseits ist die Ablehnung der Menschen- 
rechte von Seiten der Kirche ein deutlicher Hinweis darauf, dass die Menschenrechte einen viel komplexeren Entwicklungsprozess genommen haben als eine einfache Weiterentwicklung und Kodifizierung des christlichen Würdebegriffs. Hans Joas schlägt daher vor, eher von einer „Charismatisierung der Person“ zu sprechen, um aufzuzeigen, wie vielschichtig der Prozess war, der zu dem Status quo führte, indem wir den Menschen selbst als „das Heilige“ definieren und indem er den Begriff der „Sakralisierung der Person“ von Émile Durkheim aufgreift (Hans Joas 2011, 62).

Im Folgenden soll eine Konzentration auf die inhaltlichen Aspekte erfolgen, mit denen Hans Joas die unproduktive Dichotomie der beiden oben genannten Ansätze zu überwinden versucht und die mir besonders geeignet erscheinen, um der Frage nachzugehen, ob das Verständnis der Person als des Heiligen eine Auswirkung auf das Verhältnis zwischen Menschen und Tieren hat - sei es positiv im Sinne einer Verpflichtung des Menschen auf den Schutz seiner Mitgeschöpfe oder negativ im Sinne einer argumentativen Stützung der Unterdrückungsgeschichte der Tiere durch den Menschen. Dafür möchte ich zwei Aspekte im Verständnis der Person bei Hans Joas aufgreifen, nämlich seine Beschäftigung mit der Frage nach der "Seele und dem Selbst“ und die Auffassung, das Leben als „Gabe“ zu verstehen.

\subsection{Der Weg von der „Seele zum Selbst“: eine Brücke zu einer umfassenden Relationalität}

In der Tradition des amerikanischen Pragmatismus beschäftigt sich Hans Joas mit dem Verhältnis zwischen dem traditionellen Konzept der „Seele“ und dem Konzept des „Selbst“ (Hans Joas 2011, 211). So beschreibt er, wie die substanzmetaphysische "Seele“ der klassischen Theologie zum „Selbst“ wurde, das bis heute in der Soziologie und Psychologie überlebt hat. Diesen Transformationsprozess beschreibt er vor allem mit Hilfe von William James. Anders als beispielsweise bei John Dewey oder George Herbert Mead sieht er bei William James eine größere Sensibilität für den vollen Inhalt des klassischen Seelenbegriffs (Hans Joas 2011, 222). Während der Transformationsprozess ,von der Seele zum Selbst“ bei John Dewey und George Herbert Mead Teil eines intellektuellen Säkularisierungsprojekts war, ging es William James eher darum, den genuin religiösen Inhalt des Seelenbegriffs zu erhalten. In Übereinstimmung mit William James ist sich Hans Joas sehr wohl bewusst, dass er sich hier in einem Bereich jenseits der bloßen Rationalität bewegt. Vielmehr geht es darum, auszudrücken, dass der Mensch ein Wesen mit „moralischer Tiefe“ ist, das auf Rela- 
tionalität und Kreativität angewiesen ist. Auch wenn Hans Joas dies nicht ausdrücklich sagt, gibt es hier eine ethische Dimension: Der Mensch kann seine Freiheit im Guten wie im Schlechten verwirklichen. Er ist ein Wesen der Sakralität, gerade weil er zur Freiheit bestimmt ist und sich selbst transzendieren kann. An dieser Stelle muss auch der Einwand der französischen Philosophin Simone Weil thematisiert werden, die mit aller Schärfe darauf hingewiesen hat, dass die Rede von der sakralen Person niemals in einer Abstraktion enden darf, sondern immer den Menschen in seiner Konkretheit vor Augen haben muss (Simone Weil 2018, 18).

Wenn die Sakralität der Person als des konkreten und geschöpflichen Menschen in diesem Sinne nicht als monadische Abgeschlossenheit in einem für alle anderen unzugänglichen Bereich des fanum verstanden wird, sondern sich in Relationalität verwirklicht, dann darf auch ein umfassenderer Begriff von Relationalität behauptet werden, der den Menschen nicht auf seine menschlichen Kontakte beschränkt. Diese Form einer auch seine natürliche Umwelt umfassenden Relationalität des Menschen würde dann bedeuten, dass seine Verfasstheit als beseelte Person ihn in die Lage versetzt, auch zu tierischen Mitgeschöpfen in ein Resonanzverhältnis zu treten und so einen Beitrag zur Überwindung der Entfremdung zwischen Menschen und Tieren zu leisten, die dann nicht mehr aufgrund einer mangelnden substanzmetaphysisch gedachten Seele abgewertet werden müssten, sondern als Gegenüber für den auf Relationalität angelegten sakralen Wesenskern des personalen Selbst des Menschen in ihrer Andersheit wertgeschätzt werden könnten. Hier liegt m. E. auch das Potenzial für eine Überwindung der aus Sicht der Tiere unheilvollen Diskussion um die Beseelung von nicht-menschlichen Lebewesen. Wenn das menschliche Selbst in seiner relationalen Ausrichtung begriffen wird, können vermeintlich nicht-beseelte Lebewesen nicht mehr utilitaristisch nur durch ihre Relevanz für den Menschen beurteilt werden. Vielmehr werden sie eingebunden in einen ganzheitlichen Resonanzzusammenhang des Menschen mit seiner Umwelt, der eine „Ahnung von einer tiefen Verbundenheit“ (Hartmut Rosa 2019, 317) in sich birgt, ohne jedoch die nicht-menschliche Welt gänzlich verfügbar und damit allein zum Objekt menschlicher Kontrolle zu machen.

\section{2 „Das Leben als Gabe“: das kenotische Element des Lebens in einer umfassenden Liebesdynamik}

Als zweiten Punkt im Verständnis des Menschen bei Hans Joas, der für eine tierethische Weiterführung in der theologischen Ethik besonders ge- 
eignet erscheint, möchte ich auf die Idee des Lebens als Gabe eingehen (Hans Joas 2011, 232-250). Hier greift Hans Joas einen Begriff auf, den er der christlichen Schöpfungstheologie entnimmt und neu überdenkt. So soll die Idee des Lebens als Gabe Gottes der bloßen Faktizität des Da-Seins gegenübergestellt werden - gleichsam als qualitative Dimension der Geschöpflichkeit, vergleichbar etwa mit der klassischen Unterscheidung zwi-

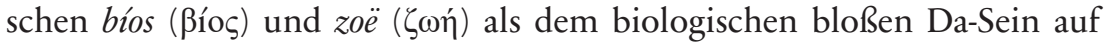
der einen und der moralisch qualifizierten Existenz auf der anderen Seite. Näherhin wird dieses Leben als Gabe Gottes qualifiziert durch das Ethos der Liebe (Hans Joas 2016, 89), welches im Sinne eines augustinischen Liebesbegriffes, wie ihn schon Hannah Arendt herausgearbeitet hat (Hannah Arendt 2018, 97), als sich zu bewährende Tugend im vorpolitischen Raum verstanden werden kann. Das bedeutet, dass die Liebe ein supra-moralisches Ordnungsprinzip über den moralischen Kategorien der Organisation des gesellschaftlichen Lebens wie etwa der Gerechtigkeit ist. In Bezug auf die Reziprozität, wie sie etwa in der Gabe-Theorie von Jacques Derrida (Jacques Derrida 1993, 93-137) analysiert wird, bedeutet dies, dass aus dem Verständnis des Lebens als Gabe niemals eine berechnende Haltung folgen darf und damit der „Monetarisierung der Beziehungen“2 Einhalt geboten werden muss. Dieser Verweis auf die supra-moralische Dimension der Liebe soll die Verpflichtung zur Gerechtigkeit nicht auflösen. Das Liebesethos sollte durch seinen Status als supra-moralische Dimension, also als Prinzip über den Dingen der Moral, verstanden werden. Was jedoch aus dem Leben als Gabe abzuleiten ist, ist eine Verpflichtung zum Schutz des Menschen, wie Hans Joas sagt, die sich - nicht auf bloßer Gegenseitigkeit beruhend - als moralische Verpflichtung aus dem empfangenen Geschenk ergebe, das allen Menschen gleichermaßen und unverdient zukomme. Vor allem der Aspekt der nicht zwingenden Reziprozität ist m. E. besonders auch für das Verhältnis des Menschen zu den Tieren fundamental und besonders anschlussfähig für eine ethische Bestimmung der Mensch-Tier-Beziehung. Der bewusste Verzicht auf die Reziprozitätserwartung in der Mensch-Tier-Beziehung kann dann als eine Überwindung der Logik des Marktes und der Ökonomisierung - die Papst Franziskus als Monetarisierung der Beziehungen bezeichnet - verstanden werden. Das durch das Liebesethos qualifizierte Leben, das als Gabe verstanden wird, befähigt den Menschen, selbst zu einer Gabe zu werden und Schutzräume des Lebens

2 So etwa Papst Franziskus in seiner Ansprache während des interreligiösen Treffens am Founder's Memorial während der Apostolischen Reise nach Abu Dhabi am 4. Februar 2019. 
für andere Geschöpfe zu schaffen. Die Erkenntnis des eigenen Verdanktseins kann somit in eine bewusste Haltung oder ein „Lebenswie“ (Clemens Sedmak 2017, 6) einführen, das ein kenotisches Element beinhaltet: Besonders in der Hinwendung zu Geschöpfen, die dem allgemeinen Vernehmen nach „unter dem Menschen“ stehen, beweist sich die lebenspraktische Konsequenz, die aus dem Leben als Gabe erwächst, indem der Mensch Lebensmöglichkeiten und Schutzräume für jedwedes Leben fördert und bewahrt. Diese kenotische Liebesdynamik verlangt demnach auch eine Hinwendung zu allen Geschöpfen, die mit dem Menschen die Eigenheit eines als Gabe des Schöpfers verstandenen Lebens teilen.

\subsection{Die Sakralität der Person und die Achtung allen Lebens als affektiver Wert}

Mit seiner Theorie versucht Hans Joas, die unproduktive und historisch falsche Dichotomie der beiden oben genannten Erzählungen über die Entstehung der Menschenrechte aufzulösen und stattdessen davon zu sprechen, dass die Kodifizierung dieser Rechte das Produkt eines tiefen Wertewandels ist (Hans Joas 2013, 17). Durch diesen Wertewandel hat der Mensch eine Position erlangt, die er nie zuvor hatte und die nur als sakral bezeichnet werden kann. Hier muss auch gesagt werden, dass Hans Joas sich sehr wohl der Tatsache bewusst ist, dass diese Sakralisierung immer durch alternative Sakralisierungen gefährdet ist, z. B. wenn eine Nation oder ein Volk sakralisiert wird (Nationalismus/Faschismus) oder eine soziale Klasse (Sozialismus/Kommunismus) (Hans Joas 2011, 101-102).

Zum besseren Verständnis soll daher noch eine kurze Bemerkung zur Definition der Werte bei Hans Joas eingebracht werden: Für Hans Joas (und auch für William James) erhalten Werte ihre Relevanz durch Erfahrung und entziehen sich damit letztendlich der rein rationalen Argumentation. Mit Max Scheler spricht Hans Joas davon, dass es bei der Entstehung von Werten eher darum geht, etwas zu finden, das ,an sich und für mich" (Hans Joas/Robert Spaemann 2018, 73) ist. Nach Hans Joas entstehen Werte durch eine affektive Bindung an etwas, das mir allgemein einsichtig und für mich persönlich verbindlich geworden ist, aber über eine reine Rationalität hinausgeht. Das ist der theoretische Hintergrund, vor dem Hans Joas auch die Sakralisierung der Person verortet: dass der Mensch etwas Unverfügbares ist, das in keiner Form verletzt werden darf. Für Hans Joas ist dies genau ein solcher Wert, der anlässlich einer konkreten historischen Gelegenheit relevant geworden und in kodifizierten Rechtstexten wie den Menschenrechtserklärungen festgehalten worden ist. Indem er den Ursprung des Wertes der Heiligkeit der Person nacherzählt, 
macht Hans Joas darauf aufmerksam, dass es sich um einen historisch kontingenten Prozess handelt, der immer in Gefahr ist, dekonstruiert zu werden oder an Bedeutung zu verlieren, sofern der Wert der Heiligkeit der Person nicht mehr geteilt wird. Es geht ihm also vor allem darum, eine affirmative Alternativerzählung anzubieten, in der die menschliche Person als das Heilige dargestellt und damit als universell schutzbedürftig charakterisiert wird (Hans Joas 2011, 147). Wie bereits erwähnt kann eine reduktionistische Sicht der menschlichen Person durchaus als relevant für die Tierethik angesehen werden. Durch die Sensibilität für die historische Kontingenz des Konzeptes der Sakralität der Person, wie sie bei Hans Joas vertreten wird, kann aber auch gesagt werden, dass die Auswirkung dieses Wertes nicht zwingendermaßen allein auf menschliches Leben fixiert bleiben muss. Die ethische Relevanz des nicht-menschlichen Lebens, die im akademischen Diskurs und vor allem auch in der Alltagskultur (Umweltschutz, Tierschutz) immer stärker wahrgenommen wird, deutet eher darauf hin, dass die Bindung an den Wert der Sakralität der Person eine Ausweitung hin zu einer allgemeinen „Ehrfurcht vor dem Leben“ (Albert Schweitzer 2013) - um einen maßgeblich von Albert Schweitzer geprägten Begriff zu gebrauchen - oder in der Terminologie von Hans Joas einer "Charismatisierung“ des Lebens erfahren kann. Diese Ausweitung geht jedoch nicht zwangsläufig auf Kosten der Hochachtung vor dem besonderen Wert der menschlichen Personalität.

\section{Sakrale Person-profanes Tier? Ausblick auf eine theologisch-ethische Verhältnisbestimmung im Ausgang vom Konzept der Sakralität der Person}

Wie durch die Nachzeichnung des Gedankenganges von Hans Joas deutlich geworden ist, wird einzig der Mensch als Person, noch dazu als sakralisierte Person verstanden. Ansätze wie beispielsweise von Peter Singer, den Personbegriff auch auf das Tierreich auszuweiten (Peter Singer 1993, 55-83), sind mit diesem Ansatz unvereinbar, da der Personbegriff nicht an einigen Fähigkeiten, über die u. U. auch Tiere verfügen können, festgemacht wird. Vielmehr wird die Person über die transzendentale Fähigkeit des Menschen definiert, sich in ein Verhältnis zu sich selbst und zu seiner Natur zu setzen. Mit anderen Worten: Der Mensch hat Personalität qua seiner Fähigkeit zur freien sittlichen Entscheidung und ist - theologisch gesprochen - das einzige Wesen, das zum Objekt einer göttlichen Offenbarungswirklichkeit und eines damit verbundenen sittlichen Anspruchs wird. Diese Existenzweise als Anspruch und Gabe zu verstehen, die den Menschen in eine kenotische Liebesdynamik einführt, entbehrt letztlich 
einer utilitaristischen Nutzensteigerung und Reziprozitätserwartung, die mit der augustinischen Terminologie als uti bezeichnet werden könnte (Peter Schallenberg 2019, 140). Die Teilhabe am universalen Liebesethos, zu dem der Mensch als relationales und personales Wesen berufen ist, kann in diesem Kontext als ethischer Grundentscheid gedeutet werden, der jeder sittlichen Einzelentscheidung fundamental vorgeordnet ist. Allgemein gesprochen bedeutet dies, dass der Mensch, der sich in seiner Personalität als sakrales Wesen versteht und damit in den Gabe-Charakter des Lebens einstimmt, von seinem Zerstörungspotenzial keinen Gebrauch macht und stattdessen - wieder mit Augustinus - in eine Logik des frui eintritt, also des wohlwollenden und des genießenden Lebensvollzugs seiner selbst und seiner Mitwelt, der ein quasi „paradiesisches“ Wachstumsrecht zugebilligt wird. Diese Haltung ermöglicht es dem Menschen, der als einziges Wesen Subjekt sittlicher Handlungen sein kann, die ihn umgebende nicht-menschliche Welt, sei sie belebt oder unbelebt, als Objekt und Adressat in seine ethischen Reflexionen einzuschließen. Der konkrete sittliche Anspruch an den Menschen als sakraler Person besteht dann zunächst in dem Streben nach einer umfassenden größtmöglichen Gerechtigkeit, die alle Geschöpfe in die Güterabwägung mit einbezieht. Diese Gerechtigkeitsforderung betrifft dann einerseits die individualethische Güterabwägung beispielsweise in der Entscheidung für oder gegen eine vegetarische oder vegane Ernährung oder im individuellen Kaufverhalten. So müsste z. B. die individualethische Güterabwägung nach den Kriterien der Relationalität, die auch die nicht-menschliche Umwelt umfasst, ein negatives Urteil fällen über Produkte, die offensichtlich auf der Grundlage einer reinen Verzweckung und Ökonomisierung von Tieren hergestellt werden. Andererseits wird dadurch auch die sozialethische und politische Dimension berührt, etwa in den Fragen von artgerechter Tierhaltung und industrieller Lebensmittelproduktion. Aus Sicht der theologischen Ethik kann dieses Ziel einer umfassenden Gerechtigkeit jedoch nur unter Berücksichtigung der eschatologischen „Makrovision“ gelingen, in der nicht nur menschliche Erlösung angestrebt wird, sondern ein umfassender Begriff von Heil, wie er schon biblisch etwa in der Vision des Lammes, das "gemeinsam mit dem Löwen“ weidet (Jes 11,6), anklingt und im Ausgang von Hans Joas im Ethos der Liebe bzw. in der supra-moralischen Dimension der Liebe mitgedacht werden kann. Die endzeitliche Vision einer die ganze Schöpfung umfassenden Aussöhnung und Erlösung kann zwar nicht bruchlos als ethischer Maßstab für die Gegenwart dienen. Zumindest soll jedoch an dieser Stelle die ethische Relevanz der Erwartung angedeutet werden, dass die gegenwärtige menschliche Zeit auch mit ihren als negativ 
erfahrenen Umständen endlich ist und auf die „Zeit Gottes“ zuläuft (Johanna Rahner 2010, 46-57).

Dass Hans Joas die Sakralisierung der Person als historisch kontingenten Prozess versteht, kann einer solchen gemäßigt-anthropozentrischen Tierethik als Mahnung gelten: die axiomatische Festschreibung der Sakralität der Person (etwa in den Menschenrechtserklärungen oder auch in Artikel 1 des deutschen Grundgesetzes) kann in diesem Zusammenhang als notwendig gewordenes Bekenntnis zur moralischen Tiefendimension des Menschen verstanden werden. Biblisch ausgedrückt könnte man sagen, dass die immer wieder neu zu artikulierende Schutzbedürftigkeit und Heiligkeit des menschlichen Lebens eine Folge des Verlustes der Gottunmittelbarkeit sind, wie sie schöpfungstheologisch eigentlich gemeint ist und wie sie in der Tierwelt - so darf vermutet werden - noch anzutreffen ist (Rainer Hagencord 42009, 33). Die Personalität des Menschen als Wesen mit "moralischer Tiefe“ als unverfügbar und unantastbar - und somit als sakral - immer wieder herauszustellen, ist da nur die axiomatische Folge eben dieses Mangels, der in existenziellen Gewalt- und Vernichtungserfahrungen gleichsam ex negativo zur Grundlage der Notwendigkeit des Bekenntnisses zur Sakralität der Person geworden ist, um so die Bedingungen eines guten und umfassend geglückten Lebens zu schützen. Die Vision eines guten Lebens für den Menschen und seine Mitgeschöpfe führt konsequenterweise in eine Haltung der Offenheit für eine universale Gemeinschaft, wie sie auch von Papst Franziskus in seiner Enzyklika Laudato si' thematisiert wird:

„Wenn das Herz wirklich offen ist für eine universale Gemeinschaft, dann ist nichts und niemand aus dieser Geschwisterlichkeit ausgeschlossen. Folglich ist es auch wahr, dass die Gleichgültigkeit oder die Grausamkeit gegenüber den anderen Geschöpfen dieser Welt sich letztlich immer irgendwie auf die Weise übertragen, wie wir die anderen Menschen behandeln. Das Herz ist nur eines. Die gleiche Erbärmlichkeit, die dazu führt, ein Tier zu misshandeln, zeigt sich unverzüglich auch in der Beziehung zu anderen Menschen. Jegliche Grausamkeit gegenüber irgendeinem Geschöpf widerspricht der Würde des Menschen. Wir können uns nicht als große Liebende betrachten, wenn wir irgendeinen Teil der Wirklichkeit aus unseren Interessen ausschließen“ (LS 92).

Dass die Mensch-Tier-Beziehung einen so prominenten Einzug auch in die kirchliche Lehrverkündigung gefunden hat, erscheint zunächst begrüßenswert. Jedoch schwingt auch in der zitierten Passage immer noch die kantianische Hypothek mit, dass das Tierwohl vor allem deshalb nicht verletzt 
werden soll, damit der Mensch nicht verroht und infolgedessen anderen Menschen schadet. Denkt man die Gabe-Theorie des Lebens und das Verständnis der Person als relationalem Wesen der Freiheit aber konsequent weiter, so verbietet sich diese verzweckende Sicht auf das Tierwohl. Vielmehr muss die Rede von der Sakralität der Person unter dem Leitmotiv des Liebesethos gedacht werden, das es ermöglicht, jede Kreatur um ihres Eigenwertes willen nicht nur zu respektieren, sondern den Mangel an relationaler Reziprozität als ethischen Anspruch an sich selbst zum Schutz der Mitgeschöpfe zu verstehen. Die Selbstzuschreibung des Menschen, dass seine Person etwas Heiliges sei, ist dann quasi als pädagogisches Moment zu verstehen, welches zu einer „Herzensbildung“ für den Bereich der Unverfügbarkeit des Lebens beizutragen vermag. Wie es um die Bildung dieses „offenen Herzens“, wie Papst Franziskus es nennt, bestellt ist, kann an der Empathiefähigkeit für jedes Lebewesen abgelesen werden, das der menschlichen Person keine Reziprozität entgegenbringen kann. Diese Fähigkeit der Einfühlung, die Ausdruck der positiven Aktualisierung der menschlichen Personalität ist, kann sich dabei durchaus schon auf biblische Denkmuster berufen: „Der Fromme kennt das Fühlen seiner Tiere, des Bösen Seele aber ist grausam“ (Spr 12,10).

Die eingangs erwähnten Tendenzen zu einem reduktionistischen Menschenbild, die sich nicht allein auf das menschliche Selbstbild, sondern auch auf sein Denken von der übrigen Schöpfung auswirken, werden durch die Neuartikulation des Wertes der Person als des Sakralen kritisiert und können in ihrer Wirkung eingegrenzt werden. Die intellektuelle und praktische Absage an eine von der Logik des Marktes durchdrungene Lebenswelt leistet so ihren Beitrag einerseits zu einer Humanisierung des öffentlichen Raums und des menschlichen Zusammenlebens. Andererseits verbietet sich gemäß dieser Logik aber auch eine Sicht auf die Tiere, in der diese in cartesianischer Diktion nur als „Automaten“ wahrgenommen werden. Es wäre ein wünschenswerter Mehrwert des Konzeptes der Sakralität der Person, wie es vor allem von Hans Joas in den Diskurs eingebracht worden ist, wenn die schöpfungstheologische gemeinsame Ursprünglichkeit aller Lebewesen als ethischer Auftrag an den Menschen in seiner Personalität stärker im theologisch-ethischen Diskurs gewürdigt würde und es dadurch gelingen könnte, die alte Furcht vor einer Minderung des Stellenwerts des Menschen durch eine Aufwertung der Tiere in der Ethik zu dämpfen. Ein theologischer Sakralismus der menschlichen Person auf Kosten der Tierwelt ist demnach nur um den Preis einer fahrlässigen Verzerrung des Konzepts der Sakralität der Person zu rechtfertigen. Der alte Vorwurf des Celsus an die Christen, dass sie den Menschen zu scharf von seiner nicht-menschlichen Umgebung trennen (Larry Siedentop 2014, 77), 
kann so gesehen auch im Kontext der Sakralität der Person keinen Bestand haben, auch dann, wenn die Entfremdungsgeschichte des Menschen von seiner nicht-menschlichen Umwelt in all ihrer Tragik berücksichtigt wird. Vielmehr muss betont werden, dass die Sakralität der Person in ihrer Tiefendimension nur dann ihr volles ethisches Potenzial aktualisiert, wenn der Mensch sich selbst als Gabe versteht und Zeugnis gibt für eine Ethik des Lebens (Eberhard Schockenhoff 2009, 561) ${ }^{3}$ in verdankter Existenz und als moralisches Subjekt, das auch seine nicht-menschlichen Mitgeschöpfe als Objekte seiner Verantwortlichkeit um ihrer selbst willen in die ethische Güterabwägung mit einbezieht.

\section{Literatur}

Arendt, Hannah 2018, Der Liebesbegriff bei Augustin: Versuch einer philosophischen Interpretation, hg. von Frauke Kurbacher, Hamburg.

Bauer, Thomas 2018, Die Vereindeutigung der Welt. Über den Verlust an Mehrdeutigkeit und Vielfalt, Stuttgart.

Berdiajew, Nikolai A. 2018, Im Herzen die Freiheit. Das Bürgertum zwischen Sinnsuche und Selbstgeißelung, Bad Schmiedeberg.

Blanke, Fritz 1993, Unsere Verantwortung gegenüber der Schöpfung, in: Teutsch, Gotthard M. (Hg.) 1993, Umwelt - Mitwelt - Schöpfung. Texte zur Verantwortung des Menschen für die Schöpfung, Stuttgart, 9-12.

Derrida, Jacques 1993, Wenn es Gabe gibt - oder: „Das falsche Geldstück“, in: Wetzel, Michael/Rabaté, Jean-Michel (Hg.) 1993, Ethik der Gabe. Denken nach Jacques Derrida (Acta humaniora), Berlin, 93-136.

Hagencord, Rainer ${ }^{4} 2009$, Diesseits von Eden. Verhaltensbiologische und theologische Argumente für eine neue Sicht der Tiere, Regensburg.

James, William 2014, Die Vielfalt religiöser Erfahrung. Eine Studie über die menschliche Natur, Berlin.

Joas, Hans 1997, Die Entstehung der Werte, Frankfurt a. M.

Joas, Hans 2004, Braucht der Mensch Religion? Über Erfahrungen von Selbsttranszendenz, Freiburg i. Br. etc.

Joas, Hans 2011, Die Sakralität der Person. Eine neue Genealogie der Menschenrechte, Berlin.

Joas, Hans 2012, Glaube als Option. Zukunftsmöglichkeiten des Christentums, Freiburg i. Br. etc.

3 Hier reflektiert Eberhard Schockenhoff auch nochmal ausführlich den Stellenwert der Personalität in einem tierethischen Kontext im Gesamtzusammenhang der Ethik des Lebens. 
Joas, Hans 2013, Der Mensch muss uns heilig sein, in: Laux, Bernhard (Hg.) 2013, Heiligkeit und Menschenwürde. Hans Joas' neue Genealogie der Menschenrechte im theologischen Gespräch, Freiburg i. Br. etc., 14-21.

Joas, Hans 2015, Sind die Menschenrechte westlich?, München.

Joas, Hans 2016, Kirche als Moralagentur?, München.

Joas, Hans 2017, Die Macht des Heiligen. Eine Alternative zur Geschichte von der Entzauberung, Berlin.

Joas, Hans/Spaemann, Robert 2018, Beten bei Nebel. Hat der Glaube eine Zukunft?, Freiburg i. Br. etc.

Kant, Immanuel ${ }^{4} 1982$, Die Metaphysik der Sitten (1797) (Werkausgabe 8), Frankfurt a. M.

Laux, Bernhard (Hg.) 2013, Heiligkeit und Menschenwürde. Hans Joas' neue Genealogie der Menschenrechte im theologischen Gespräch, Freiburg i. Br. etc.

Rahner, Johanna 2010, Die Zeit, die bleibt. Zur messianischen Grundstruktur des Eschatologischen, in: Arens, Edmund (Hg.) 2010, Zeit denken. Eschatologie im interdisziplinären Diskurs, Freiburg i. Br. etc., 46-57.

Remele, Kurt 2019, Die Würde des Tieres ist unantastbar. Eine zeitgemäße christliche Tierethik, Kevelaer.

Rosa, Hartmut 2019, Resonanz. Eine Soziologie der Weltbeziehung, Berlin.

Schallenberg, Peter 2019, Naturrecht als Personrecht. Zum Verhältnis von Recht und Sakralität in Sicht der Theologie. Herbert Tröndle zu ehrendem Gedächtnis, in: Beckmann, Rainer et al. (Hg.) 2019, Gedächtnisschrift für Herbert Tröndle, Berlin, 137-150.

Schockenhoff, Eberhard 2009, Ethik des Lebens. Grundlagen und neue Herausforderungen, Freiburg i. Br. etc.

Schweitzer, Albert 2013, Die Ehrfurcht vor dem Leben. Grundtexte aus fünf Jahrzehnten, München.

Sedmak, Clemens 2017, Die Würde des Menschen ist unantastbar. Zur Anwendung der Katholischen Soziallehre, Regensburg.

Siedentop, Larry 2014, Inventing the Individual. The Origins of Western Liberalism, Oxford.

Singer, Peter 1993, Practical Ethics, Cambridge.

Taylor, Charles 1996, Die Quellen des Selbst. Die Entstehung der neuzeitlichen Identität, Frankfurt a. M.

Turkson, Peter 2019, Integraler Humanismus und Wirtschaftsökologie. Überlegungen aus Anlass der Amazonas-Synode (Kirche und Gesellschaft 463), Köln.

Weil, Simone 2018, Die Person und das Heilige. Über Occitanien, Gespräch mit Trotzki, Wien/Leipzig. 
